$\xi=-1$

\title{
Modelling and Simulation of the Electrical Vehicle Using Matlab and Verifying it by Driving Cycles
}

\author{
Gussan Maaz Mufti1, Mobeen Ur Rehman2 and Dr. Abdul Basit 3 \\ 1 Electrical Energy Systems Engineering \\ 2Electrical Engineering DepartmentUSPCASE-P UET \\ Bahria University Islamabad Campus \\ Peshawar, Pakistan I \\ slamabad, Pakistan \\ gussan.mufti@uetpeshawar.edu.pk \\ 3Electrical Energy Systems EngineeringUSPCASE-P UET \\ Peshawar, Pakistan \\ *Corresponding author E-mail: abdul.basit@uetpeshawar.edu.pk
}

\begin{abstract}
Modelling of the Electric Vehicle is analyzed in this paper to evaluate the performance and range of the EV. The indigenous platform of MATLAB has been used to model and simulate the different scenarios of range covered by the EV by varying the accessory power associated with them. The mathematical system was further verified by simulating the standard drive cycles for different regions of the world including the standard drive cycle of U.S.A. and E.U. These results show that the power of the accessories associated with the Electric Vehicles plays a major role in deciding the distances covered by it. A suitable selection can help to increase the range of the Electric Vehicles.
\end{abstract}

Keywords: Batteries, Electric Vehicles, FUDS, SFUDS, USO6 Drive Cycle, Mathematical model, WLTC drive cycle, Matlab.

\section{Introduction}

The usage of Electric Vehicles (EVs) has proportionally increased in the recent years due to the positive impacts of the EVs. These positive impacts include their usage their non-dependency on the depleting reserves of the conventional fuels and their environmental friendly emissions as compared to the air pollution caused by the usage of the conventional vehicles .Due to the recent developments of the electrical sources such as Super Capacitors (SCs), Fuel Cells (FCs) and the advancements in the conventional battery systems, the performance of the EVs has reached new heights. Due to their independency from the increasing fuel prices of the conventional fuels and their non-emission of air pollutant characteristics the modeling and control of the EVs has received a major focus in the recent years. The modeling of the EV presents many challenges as it is the combination of the many complex sub systems such as battery management systems, electrical motors and the power electronics interfaces.

One of the means for decreasing the climate change and pollution is the inclusion of the green solutions for the transportation. In this regard the year 2015 saw the global threshold of 1 million electric cars on the road exceeded, closing at 1.26 million[9].

The market shares of electric cars rose above $1 \%$ in seven countries in 2015[10]. This clearly indicates that slowly and gradually the scope of electric vehicles is increasing. Fig 1 summarizes the global electric stock 2010-2015 Norway, the Netherlands, Sweden, Denmark, France, China and the United Kingdom. Market shares reached $23 \%$ in Norway and nearly $10 \%$ in the Netherlands. Chi- na's booming electric car sales in 2015 made it the main market worldwide, before the United States, for the first time[10]. China is also home to the strongest global deployment of e-scooters and electric buses.

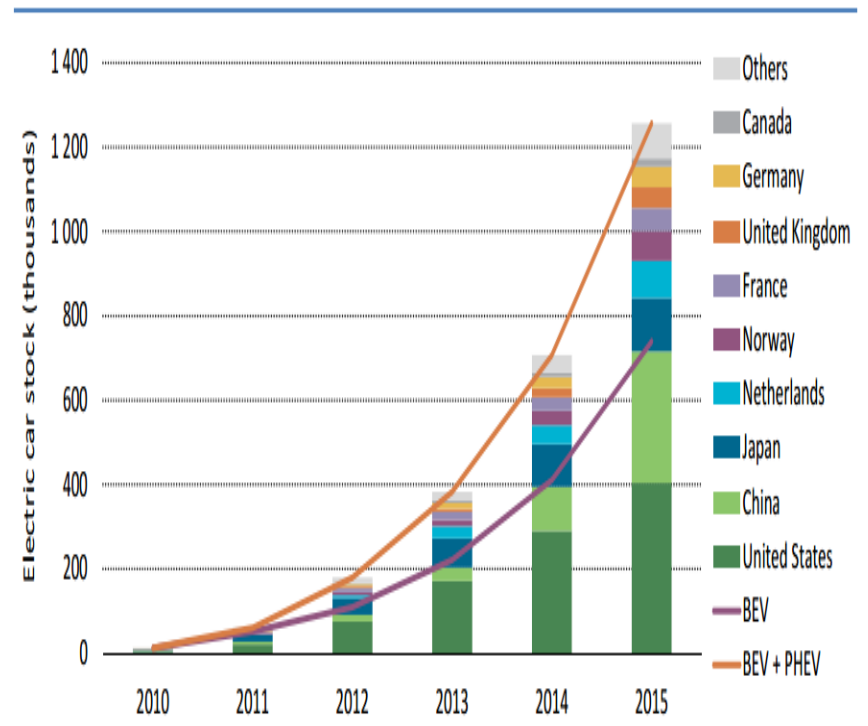

Figure 1: Evolution of Global Stock Markets2010-15[11] 


\section{Modelling and Simulation of Electrical Ve- hicles}

\section{Range Modelling and Driving Cycles}

The series modeling for the variety of electric automobiles is a most important problem. It is because of ability of the battery to resourcefully store electrical energy. At all cases, the problem is surely a serious problem in the strategy of electric automobile. There are dual categories of design or assessment that can be completed regarding the variety of an automobile. The primary, and the simplest, is the persistent speed examination. Obviously, car cannot be actually driven at continuous speed, particularly not on equal ground. Another category of test, further beneficial and multifaceted, is wherever the car is driven, with authenticity or in simulation, over an outline of ever altering velocities. These test series have been established with approximately care. The cycles are planned to link to accurate driving designs in diverse circumstances. Throughout the cycles, the speed of the automobile speed is continuously altering which consequently affects the performance, which makes the calculations more complex. Yet, recently simulation programs are able to smooth and simplify these complex circumstances into somewhat practically straightforward conditions. The concept of testing drive cycles come into play for the range simulation of the EVs. Even if the test is impractical, it can be claimed that they do at minimum give valuable relative statistics.

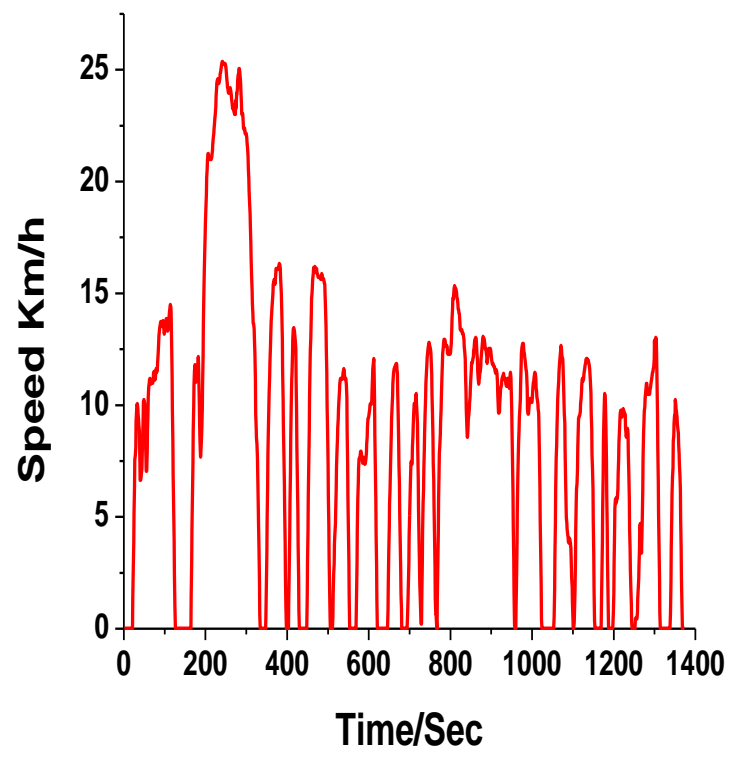

Fig 4 FUD Cycle Used for the Simulation of the EV Cycle

These driving cycles have largely been established in demand to deliver an accurate and applied test for the productions of automobiles. One of the most renowned primary cycles was constructed on real traffic movements, named as the LA -4 driving cycle. It was then transformed into Federal Urban Driving Schedule (FUDS). The duration time for this cycle is 1500 seconds, as can be seen in Figure 2. Another basic form of FUDS is SFUDS has duration of 360 seconds. It has the decrease data points but it provides very alike results like that of FUDS only the data points have been decreased. Urban driving is simulated by these cycles whereas highway driving is simulated by US06 cycle. The US06 standard is now becoming extensively used to simulate such conditions as shown in Figure 4.

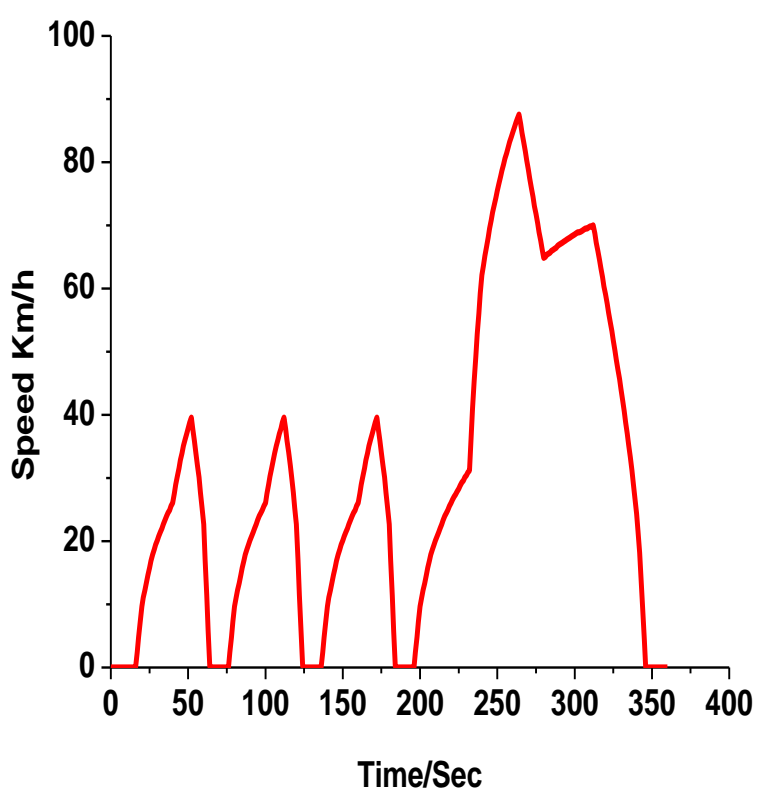

Fig 5: SFUDS Cycle Used for the Simulation of the EV Cycle

\section{Mathematical Equations for the Range Modelling of Electric Vehicle}

The Total tractive energy is provided by the following equation $F t e=F r r+F a d+F h c+F l a+F \omega a$

where the forces are calculated by the following equations

Frr $=\mu r r m g$

$F a d=1 / 2 \rho A C d v 2$

$F h c=m g \sin (\psi)$

Fla $=m a$

$F \omega a=I *(G 2 / \eta g r 2) * a$

The power required for the vehicle to move is calculated by the following equation

Pte $=$ Fte $\times v$

The inefficiencies in the controller, motor and the gear system is computed by the following equations in the acceleration mode

Pmot in $=$ Pmot out $/ \eta m$

Pmot out $=$ Ptelng

However during the DE acceleration phase the following equations are to be used

Pmot in $=$ Pmot out $\times \eta m$

Pmot out $=$ Pte $\times \eta g$

The torque is calculated by the following equation $T=P l w$

Now finding the efficiency by the following equation $\eta m=T \omega / T \omega+k c T 2+k i \omega+k w \omega 3+C$ 


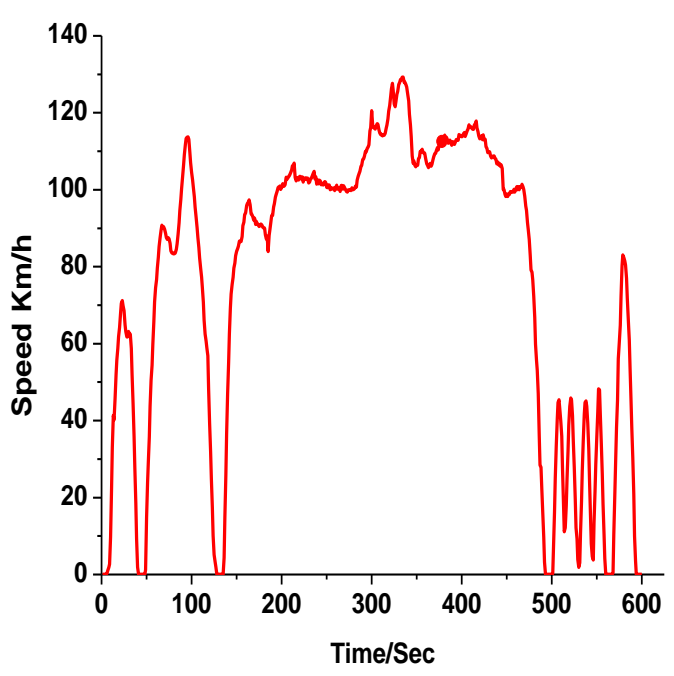

Fig. 6. High Speed (US06) Driving Cycle

Similar to earlier cycles, the Universal Consistent Light vehicles Test Procedures (WLTP) is an examination completed on EVs. It permits assessing the toxins and emissions, the fuel economy but then also the electric series of light duty cars (passenger cars as well as vans). It is industrialized by European, Japanese in addition Indian experts for replacing the NEDC cycle by 2013--2014. Class 2 cycle is representative of low, intermediate and comparatively high automobile velocities, covering Indian automobiles and European and Japanese low power automobiles. Class 1 cycle is ended of low as well as average velocity zones. It is distinctive of low power automobiles that can be originated in India.

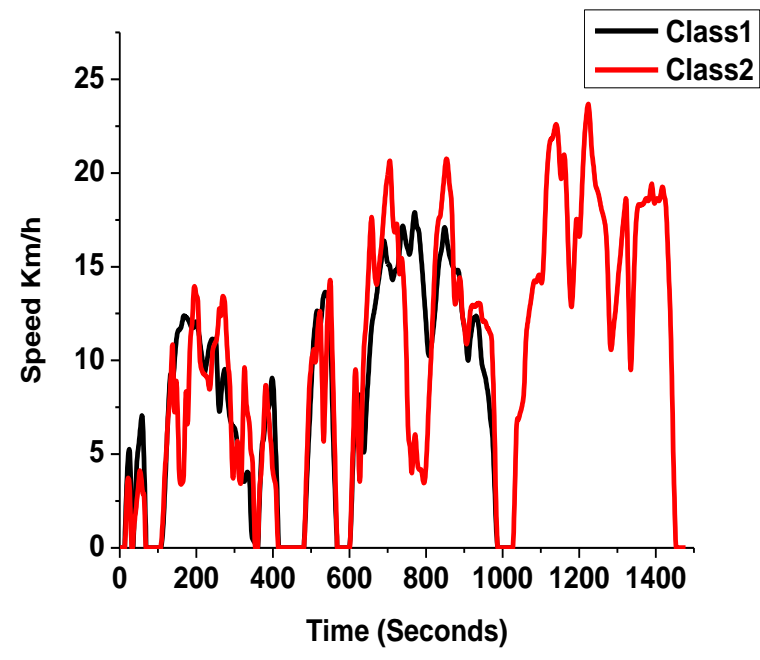

Fig. 7. WLTC cyles Class1 and Class 2

The values of the parameters depend upon the type of the electrical motor that is being used. These values can be computed by using the following table

Values for the Parameters of the EV Simulation[15]

\begin{tabular}{|l|l|l|l|}
\hline S.No. & Parameter & $\begin{array}{l}\text { Motor Parameters } \\
\text { for 2-5KW }\end{array}$ & $\begin{array}{l}\text { Parameters for } \\
\text { the Induction } \\
\text { Motor 100KW }\end{array}$ \\
\hline 1. & $\mathrm{~K}_{\mathrm{C}}$ & $1500 * 10^{-3}$ & $300 * 10^{-3}$ \\
\hline 2. & $\mathrm{~K}_{\mathrm{I}}$ & $100 * 10^{-3}$ & $10 * 10^{-3}$ \\
\hline 3. & $\mathrm{KW}$ & $100 * 10^{-6}$ & $500.0^{*} 10^{-8}$ \\
\hline 4. & $\mathrm{C}$ & $0.2 * 10^{2}$ & $6 * 10^{2}$ \\
\hline
\end{tabular}

The net power obtained from the motor can thus be calculated by using the relevant equations. However an Electrical Vehicle has also components like radio, light indicators and other accessories that also need electrical power. In order to calculate the total electrical power that would be required from the battery its necessary to add this accessory power therefore the net power computed and required from the battery is given by

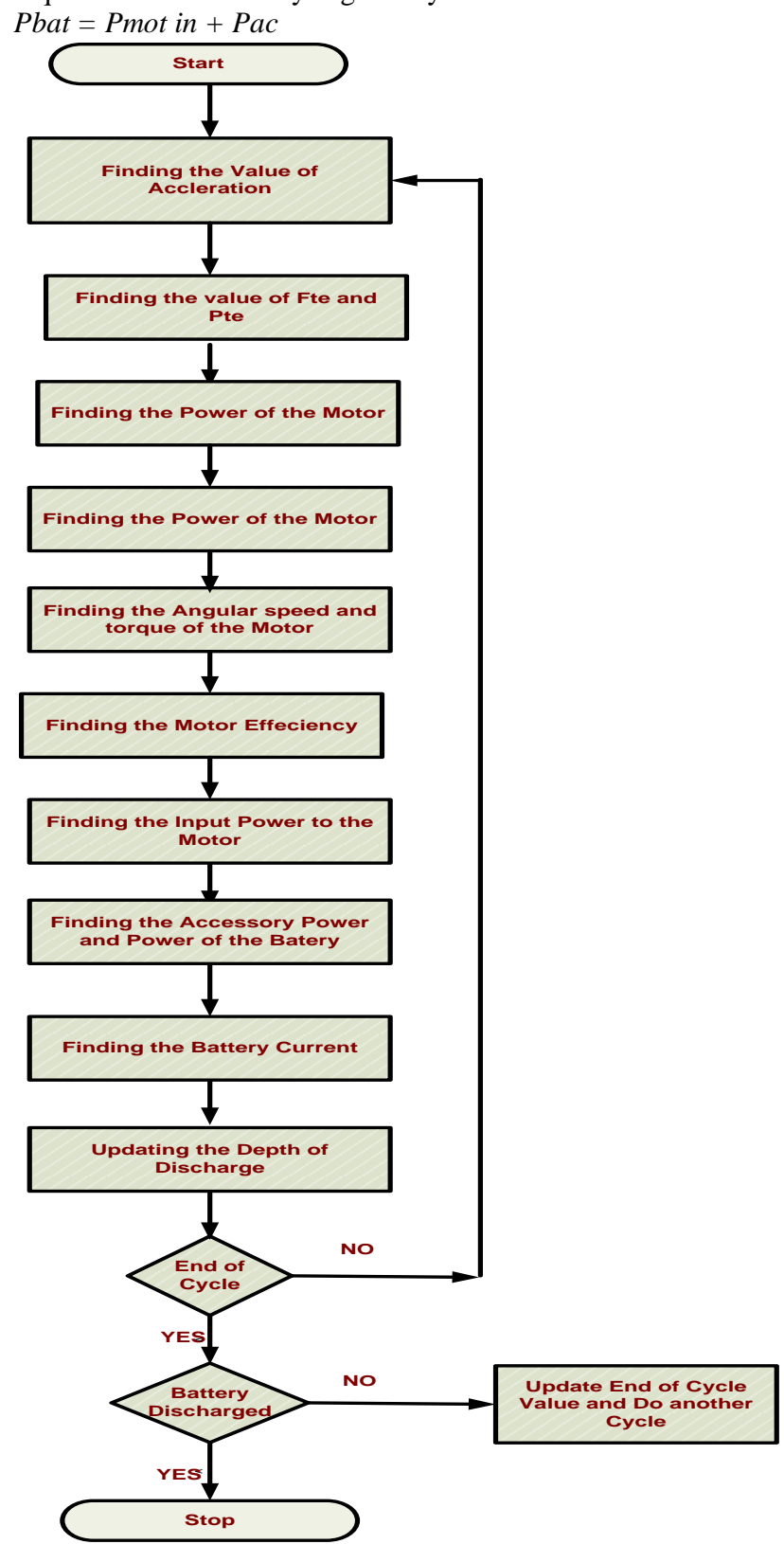

VALUES FOR THE PARAMETERS OF THE EV SimULATION[16]

\begin{tabular}{|l|l|l|}
\hline S.No. & Parameters & Values \\
\hline 1. & Frontal Area, A & $1.8 \mathrm{~m}^{2}$ \\
\hline 2. & Drag Co efficient ,Cd & 0.19 \\
\hline 3. & Mass (Battery+vehicle) & $822.5+24.49=846.9$ \\
\hline 4. & $\begin{array}{l}\text { Mass including two } \\
\text { persons }\end{array}$ & $846.9+100=946.9$ \\
\hline 5. & $\begin{array}{l}\text { Rolling Resistance Force, } \\
\text { Frr }\end{array}$ & 44.5 \\
\hline 6. & Regeneration Ratio & 0.2 \\
\hline 7. & Gear Efficiency & 0.95 \\
\hline 8. & Gear Ratio & 22.14 \\
\hline 9. & Power Accessories, Pac & $250 \mathrm{~W}$ \\
\hline 10. & Internal Resistance & $0.05 \mathrm{Ohms}$ \\
\hline
\end{tabular}




\begin{tabular}{|l|l|l|}
\hline 11. & No. of Cells & 24 \\
\hline 12. & Capacity & $150 \mathrm{AH}$ \\
\hline 13. & Pukeret Co-efficient & 1 \\
\hline 14 & No of batteries & 6 \\
\hline 15 & Capacity for each battery & $25 \mathrm{AH}$ \\
\hline
\end{tabular}

\section{Results and Discussions}

The EV was range modeled and simulated for the different driving cycle. In order to show the variance of range covered by the EV the power of the accessories was varied to the five different vales for each of the driving cycle.

It was found that the results for the FUDS and SFUDS was similar in nature as shown in fig 6 which cross checks that the cycles are same but the SFUDS is the representation of the simpler form of the FUDS. It was also found that the range of EV is maximum for the minimum accessories associated with it.

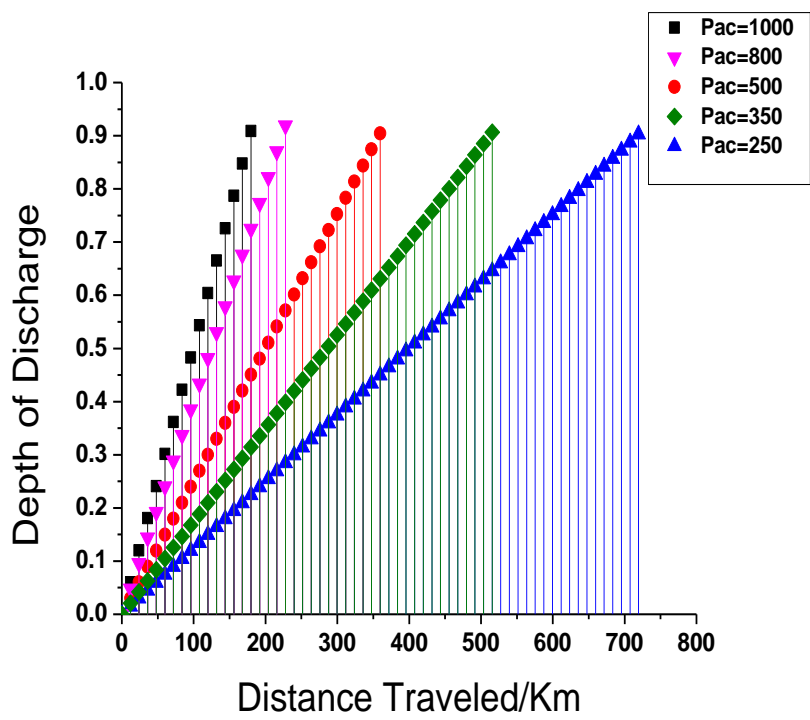

Fig 8 A graph showing the distance traveled vs depth of discharge of the design electric car for different power requirements FUDS

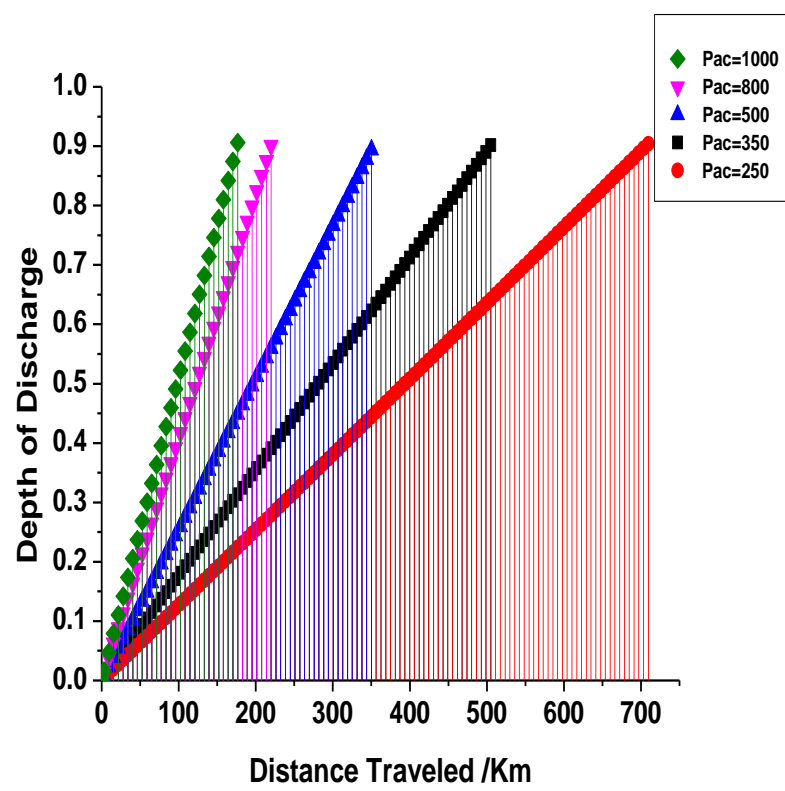

Fig 9 A graph showing the distance traveled vs depth of discharge of the design electric car for different power requirements SFUDS

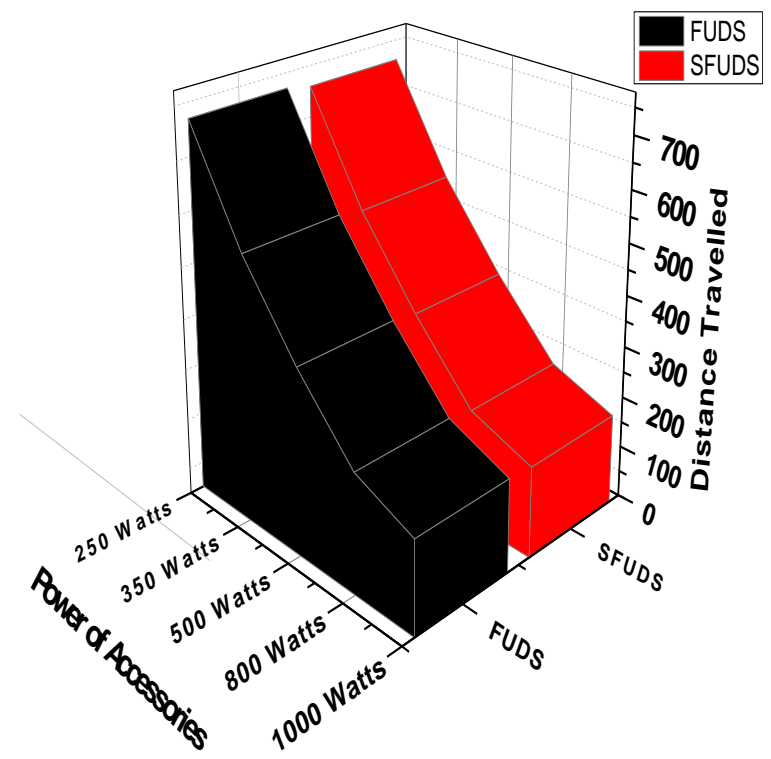

Fig 10 Comparison of the Distances Covered by FUDS and SFUDS

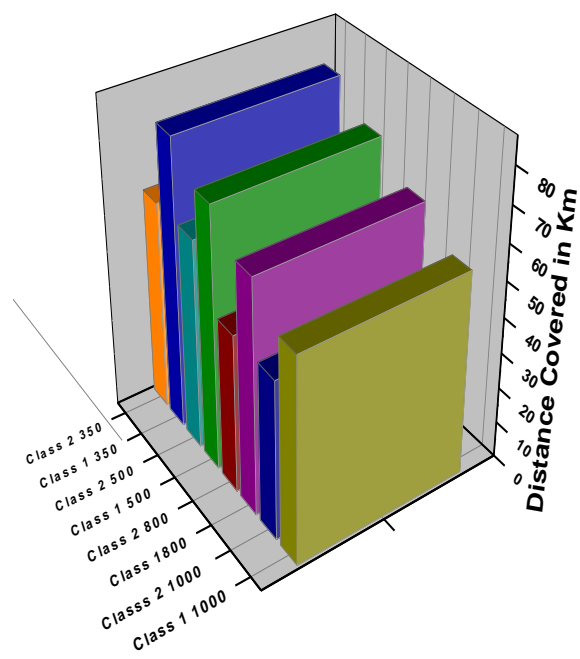

Fig 11 A graph showing the distance traveled vs depth of discharge of the design electric car for different power requirements for Class 1 and Class 2

The range of the EV significantly dropped for the class 1 and class 2 cycles which also represents the fact that the class 1 and class 2 cycles are more rigorous as compared to FUDS and SFUDS as shown in fig. 8. The same cases of the variation in the power of the accessories were repeated for the class 1 and class 2 cycle and the results were summarized for the different cases as shown in fig 8 . In a nut shell the range of the EV was always found to be more for the class 1 case as compared to the class 2 cycle since the class 1 cycle lasts for 1000 seconds as compared to the class 2 cycle which lasts for the 1600 seconds as shown in the fig 5 .

\section{Conclusion}

In this paper a classical battery powered electric vehicle has been simulated by using Matlab. The modeling of range has been carried out and been tested for driving cycles like FUD,SFUDS, Class 1 and Class 2 WLTP cycles. The simulation of these vehicles was carried out on Matlab. The possibility of applying advance 
estimation algorithms for the state of charge and calculating the greenhouse gas emissions by the particular E Zone Electric Vehicle could be the possible future work in this regard.

\section{Acknowledgment}

The authors gratefully acknowledge the comments and suggestions of the reviewers.

\section{References}

[1] ICCT, "Driving Electrification," ICCT - Int. Counc. Clean Transp. no. May, 2014

[2] D. Dineen, M. Howley, and M. Holland, "Energy in transport," Sustain. Energy Auth. Irel., 2014.

[3] D. F. O. R. Science, C. F. O. R. Information, C. Policy, R. Applications, and C. Initiatives, "DSTI / ICCP / IE ( 2007 ) 13 / FINAL Unclassified Working Party on the Information Economy," no. 2007, pp. 1-29, 2008.

[4] G. M. Mufti, M. Jamil, D. Naeem, M. U. Mukhtiar, and A. T. AlAwami, "Performance analysis of parabolic trough collectors for Pakistan using mathematical and computational models," Clemson Univ. Power Syst. Conf. PSC 2016, 2016

[5] M. S. Aziz, S. Ahmed, U. Saleem, and G. M. Mufti, "Wind-hybrid Power Generation Systems Using Renewable Energy Sources-A Review Wind-hybrid Power Generation Systems Using Renewable Energy Sources-A Review," vol. 7, no. March, 2017.

[6] S. Z. Hassan, H. Li, S. Cagriyener, T. Kamal, G. M. Mufti, M. H. Waseem, and H. Gohar, "Integration and simulation of wind with hydrogen/supercapacitor storage hybrid system," 2017 Int. Conf. Electr. Eng. ICEE 2017, pp. 1-6, 2017.

[7] A. Küçüker, T. Kamal, S. Z. Hassan, H. Li, G. M. Mufti, and M. H Waseem, "Design and Control of Photovoltaic / Wind / Battery based Microgrid System,” pp. 1-6, 2017.

[8] G. Maaz Mufti, M. Jamil, M. Nawaz, Mobeen-ur-Rehman, S Zulqadar Hassan, and T. Kamal, "Evaluating the Issues and Challenges in Context of the Energy Crisis of Pakistan," Indian J. Sci. Technol., vol. 9, no. 36, 2016.

[9] iea, "Technology Roadmap," SpringerReference, p. 81, 2015.

[10] IEA, “Energy Efficiency Market Report 2016,” IEA Publ., 2016.

[11] International Energy Agency, "Global EV Outlook 2016 Electric Vehicles Initiative," Iea, p. 51, 2016.

[12] IEA, "Energy Technology Perspectives 2016 - Executive Summary," Iea, p. 14, 2016.

[13] International Energy Agency, "Global EV Outlook 2016 Beyond one million electric cars," Iea, p. 51, 2016.

[14] S. Munnix, "E-mobility in The Netherlands The Netherlands," no. May, 2015.

[15] J. Larminie and J. Lowry, Electric Vehicle Technology Explained. 2003.

[16] "E-Zone(Electric Vehicle) Specifications." [Online]. Available: http://cttev.com/e-zone/. [Accessed: 24-Jul-2017]. 